\title{
DESIGN SECRETS AND NEW DIMENSIONS IN FIELD DRIVEN SEPARATIONS FOR MICRO TOTAL ANALYSIS SYSTEMS $(\mu$-TAS)
}

\author{
Andreas Manz \\ Imperial College of Science, Technology and Medicine, \\ Zeneca / SmithKline Beecham Centre for Analytical Sciences, \\ Dept. Chemistry, London SW7 2AY, United Kingdom
}

\section{INTRODUCTION}

Quantitative on-line surveillance of a chemical compound is usually based on sampling, sample pretreatment, including separation of mixtures, detection and interpretation of the obtained results. If all these steps are performed automatically in flowing streams, it is called a Total Analysis System (TAS). Several different types of TAS have been constructed and used for such surveillance tasks. Disadvantages of TAS for liquid samples include slow transport of a sample from the point of sampling to the location of the detection, slow separation processes, poor efficiencies of rapid separations and large consumption of fluids. Miniaturization with respect to the above mentioned criteria leads to a so called Miniaturized Total Analysis System ( $\mu$-TAS) and overcomes some of the above problems [1-3].

The recent developments in micromachining and microsystem technology influenced the modern chemistry especially by combinatorial synthesis, chemical sensors, valves and pumps. A spectacular work by Steve Terry on integration of a gas chromatograph on a silicon wafer unfortunately has not attracted sufficient attention then.

Examples of earlier work done with my group at Ciba, e.g., flow-injection analysis, continuous sample pretreatment using free-flow electrophoresis, HPLC, rapid capillary electrophoresis and synchronized cyclic capillary electrophoresis, have demonstrated the advantages and problems of $\mu$-TAS [3-9].

\section{A FEW SIMPLE RULES}

For the design of electrophoretic microstructures, it is important to follow a few rules [2]:

\section{Rules for Separation Efficiency.}

1. Principally, the absolute voltage drop obtained between the injection and detection points defines the maximum number of theoretical plates $N$ obtainable and the corresponding resolution of two neighbouring peaks $(\sqrt{N})$. The higher the applied voltage, the better.

2. For the separation of ions having a differnt charge, 100 or more theoretical plates are sufficient, but for ions of similar or identical charge, 100,000 or more theoretical plates are needed to give a reasonable resolution.

3 . Joule heating inside the capillary and the heat transport across should be kept under control. A power of less than $1 \mathrm{~W} / \mathrm{m}$ is generally no problem.

Hence, the maximum plate number $N_{\max }$ obtainable after a miniaturization of a known system is proportional to the length $L$ of the capillary divided by the diameter $d$.

$$
N_{\max } \propto \frac{L}{d}
$$

If the miniaturization involves identical factors for $L$ and $d$, there should be no loss in performance. It can be improved, however, by increasing $L$ or decreasing d.

\section{Rules for Analysis Time.}

4. The length of the capillary influences the analysis time. A few centimeters can give a few tens of seconds.

5. The efficiency of the separation column will only be visible, if contributions of injection and detection processes are small compared to volume or length of the separation column. This includes space (length, volume) and time effects (response time).

Hence, the minimum analysis time $t_{\min }$ obtainable after a miniaturization of a known system is proportional to the length $L$ of the capillary multiplied by the diameter $d$.

$$
t_{\min } \propto L \cdot d
$$


This means, the only way of increasing the speed of an analysis and keeping the efficiency constant is to reduce the diameter and the length of a capillary by the same factor.

The above considerations are used for the very simple capillary electrophoresis system. As you can see from Figure 1, there would be other forms of detection (time constant), or other types of running the electrophoresis (free-flow electrophoresis).

In most cases, a spreadsheet calculation of a model case will give sufficient information for the design of a microstructure layout. One has to bare in mind, however, that the calculated efficiency and analysis time values may vary up to a factor 2 .

\section{Capillary Electrophoresis}

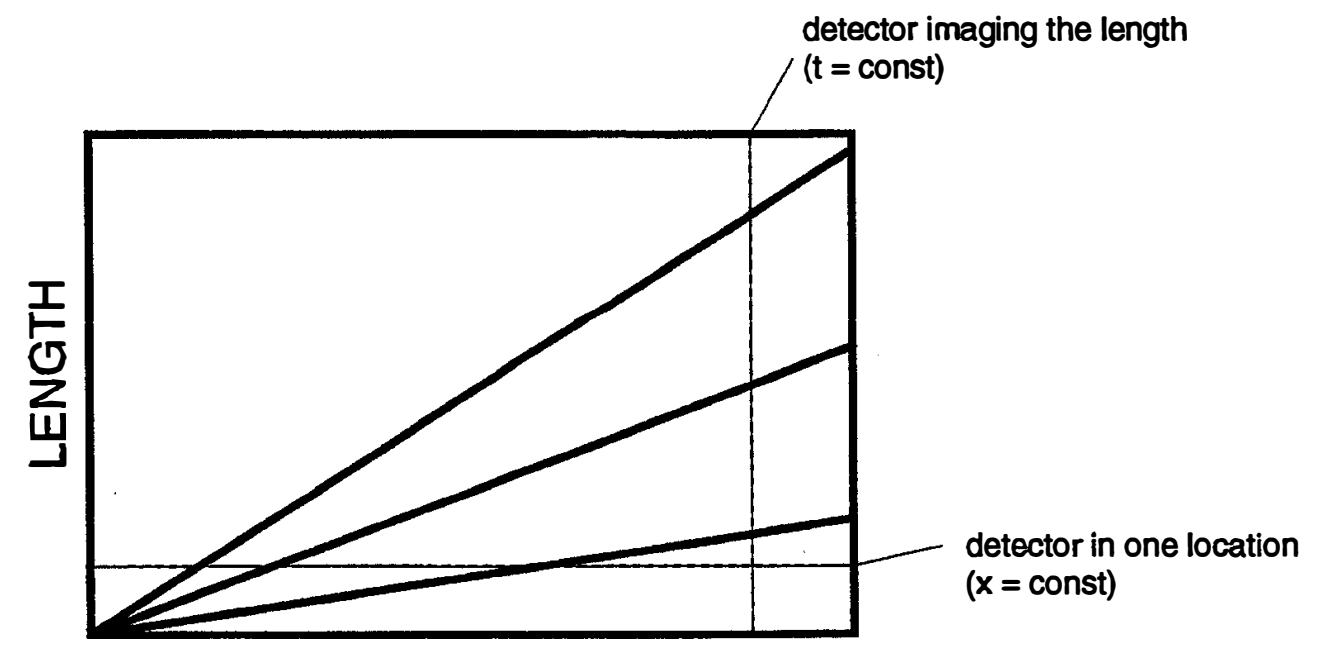

TIME

\section{Free-Flow Electrophoresis}

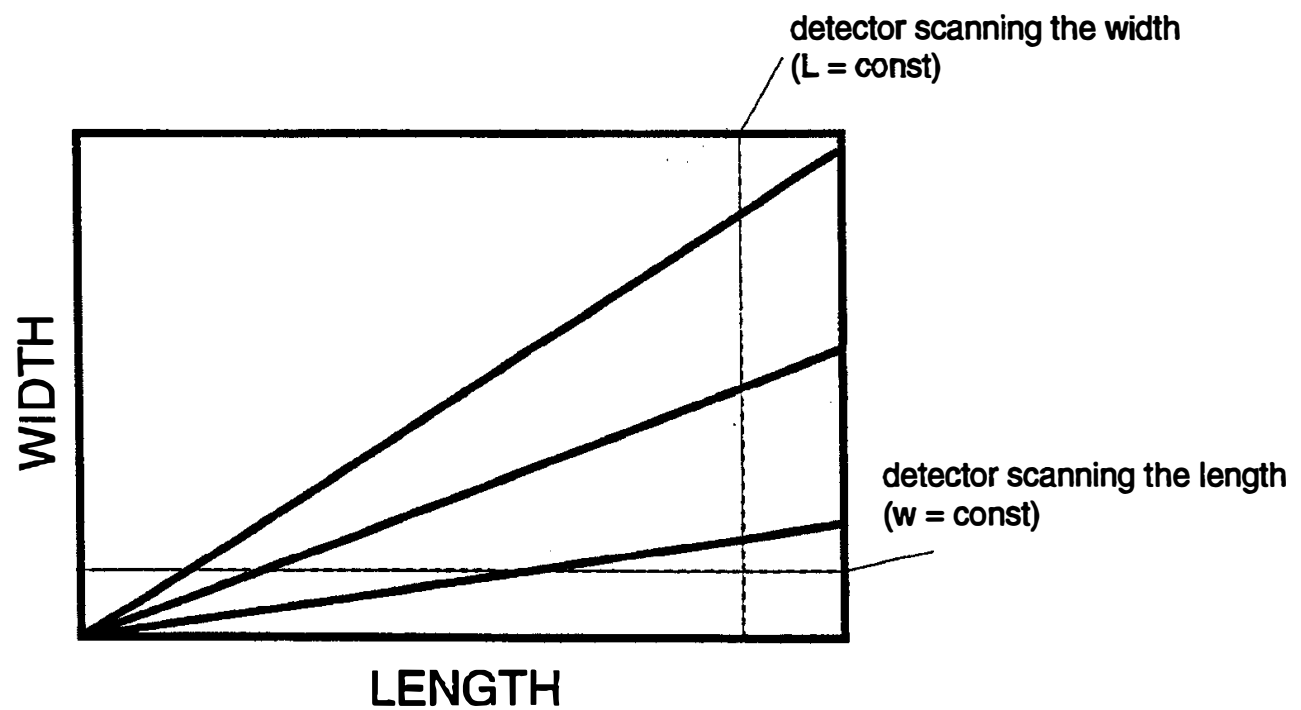

Figure 1. Comparsion of the two most common detection schemes for capillary electrophoresis (CE) and freeflow electrophoresis (FFE). 


\section{EXAMPEE: \\ FREE-FLOW ELECTROPHORESIS}

For the primary sample preparation steps in a $\mu$-TAS, continuous techniques are clearly advantageous, e.g., filtration, dialysis, SPLITT or free-flow electrophoresis (FFE). A special version of continuous zone electrophoresis in quartz channel structures has been presented by Mesaros et al. recently. The general principle of FFE is given in Figure 2. A pumped flow (carrier) in a first dimension and an electric field in a second dimension allows for the continuous separation of sample components being continuously introduced at the top of the flat separation bed. Conventional FFE is a preparative method used for the isolation of various biological components. Typical separation bed dimensions are $10 \mathrm{~cm}$ wide, $50 \mathrm{~cm}$ long and 0.4 to 1 $\mathrm{mm}$ deep. Ion exchange membranes are used to isolate the electrodes from the separation bed. FFE has been miniaturized and integrated onto a silicon micro structure by reducing all dimensions by a factor of 10 ([6], Figure 3). The ion exchange membranes have been replaced by arrays of narrow ' $V$ '-groove channels each $10 \mu \mathrm{m}$ deep and $12 \mu \mathrm{m}$ wide at the base.

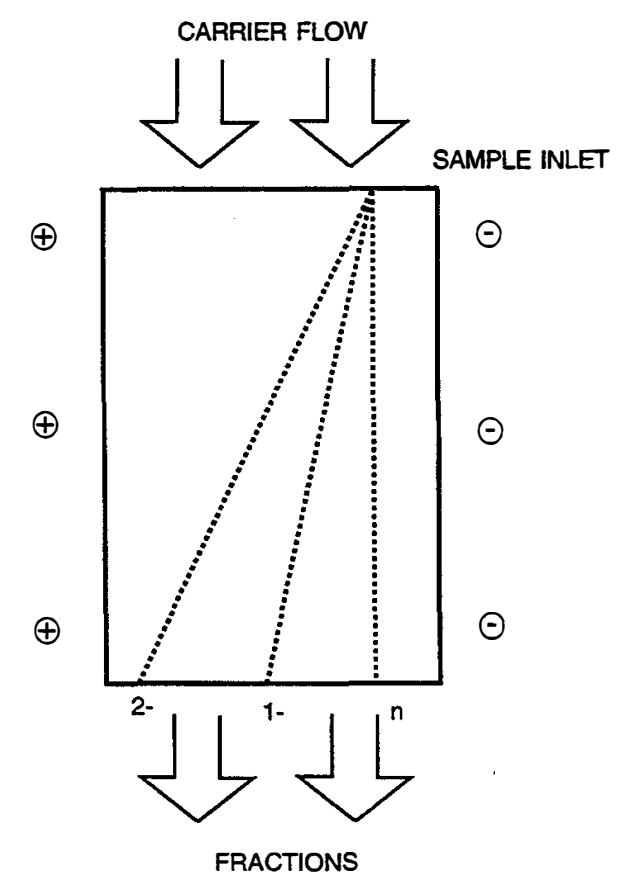

Figure 2. Schematic drawing of a FFE separation.

It is possible to achieve a continuous separation of small ions according to their charge. A voltage drop of $50 \mathrm{~V}$ gave a reasonable resolution of ions differing by one charge unit with a response time of 2 to 5 minutes (see Figure 4). The fraction of interest can be isolated and transferred to a next pretreatment step or a detection unit. Furthermore, it is possible to separate small ions from biological cells. Increased voltages should allow for higher separation efficiency and a response time of a few seconds.

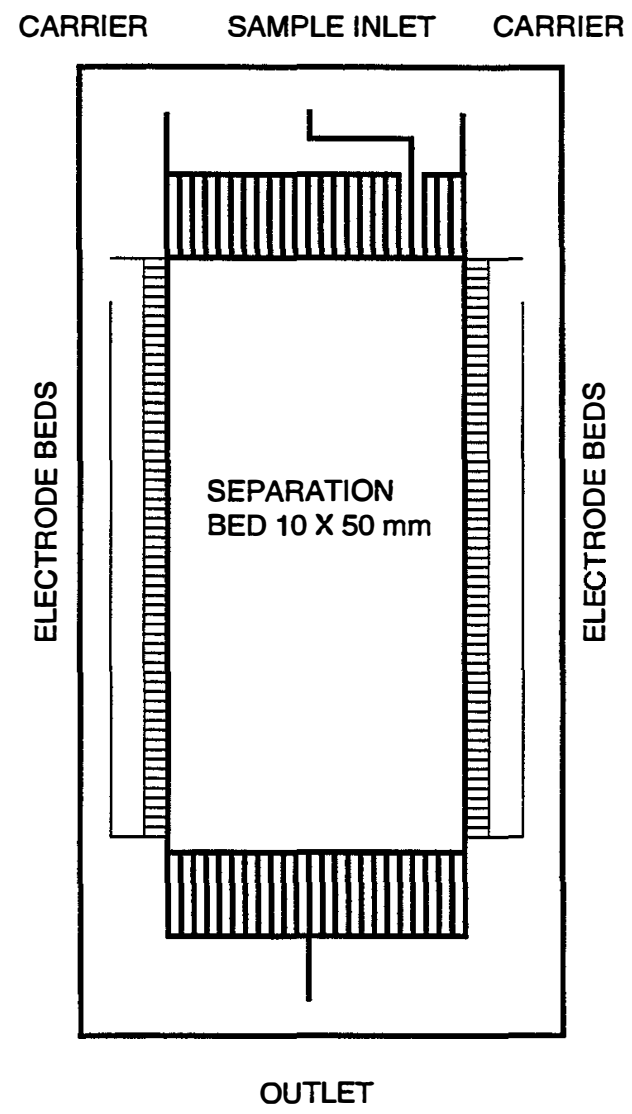

Figure 3. Layout drawing of the silicon part of a FFE separation microstructure.

\section{CONCLUSION}

For the design of electrophoretic microstructures, a few simple considerations may be very helpful to reach a performance near the desired optimum. This can be high speed, maximum performance, a compromise between the two, or a hyphenation of several separations. In the future, applications in the bioanalytical area involving molecular recognition, e.g., immunoassays or DNA hybridization, may offer a large number of opportunities which only need a defined intermediate performance. On the other hand, the electrophoretic separation as such and especially 
the two-dimensional slab gel electrophoresis can be optimized to its maximum and offers a vast separation efficiency sufficient for thousands of components in a mixture.

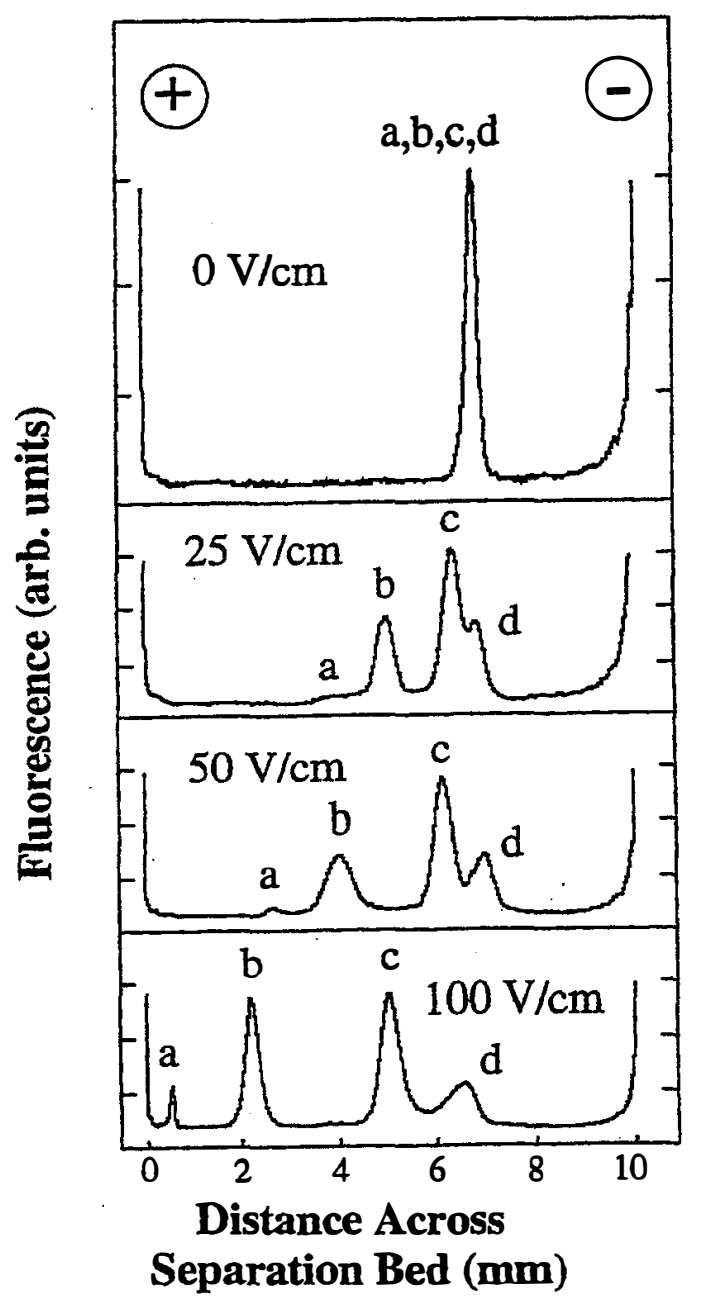

Figure 4. Separation of large biomolecules at various field strengths. Sample (a) FITC label, (b) human serum albumin, (c) bradykinin, and (d) ribonuclease A.Flow rate $15 \mathrm{~nL} / \mathrm{min}, 20 \mathrm{mM}$ borate, $100 \mathrm{mM}$ TRIS buffer ( $p H$ 9). Detection after $31 \mathrm{~mm}$, residence time 62 seconds.

\section{References}

[1] internet: http://www.ch.ic.ac.uk/manz/

[2] A.Manz, D.J.Harrison, E.Verpoorte, H.M.Widmer, "Planar chips technology for miniaturization of separation systems: a developing perspective in chemical monitoring" in Advances in
Chromatography, Vol. 33, Chapter 1, pp 22-36 (1993).

[3] A.Manz, E.Verpoorte, D.E.Raymond, C.S.Effenhauser, N.Burggraf, H.M.Widmer, " $\mu$ TAS - Miniaturized total chemical analysis sytems" in Micro Total Analysis Systems, A.van den Berg, P.Bergveld, eds., MESA Monograph, Vol. 1, Kluwer Academic Publishers, Dordrecht, p. 5-27 (1995)

[4] C.S.Effenhauser, A.Manz, H.M.Widmer, "Manipulations of sample fractions on a capillary electrophoresis chip", Analytical Chemistry 67, 2284-2287 (1995)

[5] G.Ocvirk, E.Verpoorte, A.Manz, M.Grasserbauer, H.M.Widmer, "High-performance liquid chromatography partly integrated onto a silicon chip", Analytical Methods and Instrumentation 2, 74-82 (1995)

[6] D.E.Raymond, A.Manz, H.M.Widmer, "Continuous sample pretreatment using a freeflow electrophoresis device integrated onto a silicon chip", Analytical Chemistry 66, 2858-2865 (1994)

[7] N.Burggraf, A.Manz, E.Verpoorte, C.S.Effenhauser, H.M.Widmer, N.F.de Rooij, A novel approach to ion separations in solution: synchronized cyclic capillary electrophoresis", Sensors and Actuators B20, 103-110 (1994)

[8] R.Freaney, A.McShane, T.V.Keaveny, M.McKenna, K.Rabenstein, F.W.Scheller, D.Pfeiffer, G.Urban, I.Moser, G.Jobst, A.Manz, E.Verpoorte, H.M.Widmer, D.Diamond, E.Dempsey, M.Smyth, "Novel instrumentation for real-time monitoring using miniaturised flowsystems with integrated biosensors", Annals of Clinical Biochemistry, submitted

[9] E.Verpoorte, B.H.van der Schoot, S.Jeanneret, A.Manz, H.M.Widmer, N.F. de Rooij, "Threedimensional micro flow manifolds for miniaturized chemical analysis systems", J. Micromechanics and Microengineering 4, 246-256 (1994) 